\section{Particle physics programme}

SIR-Steven Weinberg's Commentary article (Nature 330, 433; 1987) presented the prevalent view that particle physics (defined as quantum field theory, general relativity and related areas of astrophysics and cosmology) is "at a level which is in fact in absolute terms quite deep, perhaps close to the final source". This optimistic view needs to be balanced by a more critical look at current problems in particle physics.

The 'Standard Model' of particle physics has not been able to retrodict, let alone predict, the masses of quarks and leptons or to provide an explanation for the organization of particles into regular families. The mass and interaction characteristics of the hypothetical Higgs boson, a key particle, cannot be predicted. Many variants on the particle physics theme require more dimensions than the familiar four of space-time, but there is no known way to test for them. The hypothesized unification of the four forces is predicted to occur at energy levels that will probably never be accessible to experiment.

Quark confinement, charm, the 'quark-antiquark sea', gluons, the Higgs mechanism and renormalization all 'work', but they are ad hoc theoretical fixes to contradictions between theory and observation. The hypothetical magnetic monopole is another key particle in the elementary particle pantheon, yet after 40 years of assiduous searching (and readjusting of parameters) physicists are still waiting for a real detection of a magnetic monopole. One wonders if particle physicists would allow nature to answer in the negative on this crucial issue. The fundamental prediction of the Standard Model, that spin would be irrelevant to high-energy elastic scattering, has been falsified by Kirsch's scattering experiments over the past decade.

In summary, there are at least 20 basic parameters that are crucial to the particle physics programme (such as particle masses, coupling strengths, magnitudes of $\mathrm{CP}$-violations and so on) that cannot be rigorously derived and are therefore manually adjusted to agree with observations. Superstring theory, which has most particle physicists in a highly excited state, is completely untestable, for now and for the foreseeable future.

On the cosmological front, things are somewhat worse. In the standard cosmological model, the Big Bang (with or without inflation), the key events in the evolution of the Universe are supposed to have taken place within $10^{-25} \mathrm{~s}$ of the Big Bang, and thus are empirically unverifiable. None of the popular and muchtouted models predicted the existence of the dark matter, the large-scale streaming of galaxies or the existence of structures on scales larger than 300 million light years. The very existence of galaxies has never been adequately explained by any of the widely accepted theories of cosmology, astrophysics or particle physics.

One could go on, but perhaps the point has been made. The particle physics programme and its offshoots in astrophysics and cosmology are plagued by fundamental gaps, untestable assumptions and ad hoc fixes. It is possible that these problems will be overcome gradually in the coming decades, but the contention that we are on the verge of a complete unification of physics, "perhaps close to the final source", is still wishful theoretical whistling against a stiff empirical wind.

Robert L. Oldershaw

\section{West Pelham Road}

Shutesbury, Massachusetts 01072, USA

\section{Exploiting authors}

SiR-The publication of increasing numbers of multi-author scientific works may be leading to the exploitation of contributing authors. In the past couple of years, I myself have been invited to contribute to four such books, with three US publishers and one European. Each publisher offered to pay about $£ 5$ a printed page.

Some very approximate sums indicate what contributors lose by accepting such offers. Consider a book of 400 pages with 10 chapters, each with a different author, the volume costing $£ 50$. At $£ 5$ a page, each author receives $£ 200$; but were 10 per cent royalties (a usual rate for ordinary scientific books of reputable publishers) divided equitably among the authors on a sale of, say, 2,000 copies, each author would receive $£ 1,000$. Even if I have overestimated sales by a factor of two, the argument would still be valid.

Five years ago, Watkinson (Nature 300, $111 ; 1982)$ told us that most of the royalties on multi-authored works went to the editors. This arrangement is absurd as, in my experience, few editors do much editing and, indeed, they seldom earn their 5 per cent, or whatever. Although there must be some honourable exceptions, editors seldom do most of the work.

Young scientists have a special need to publish as much as they can to further their careers. They are delighted to receive any money for an article, which they would usually be glad to have accepted by an academic journal, without remuneration. So they are easy prey for the rapacious publisher. Professional institutes and learned societies might well join together to negotiate minimum rates and other conditions, such as compensation for unduly delayed publication. This would especially benefit those of their members who are inexperienced or do not have the clout to deal with commercially minded enterprises. The publishers themselves should appreciate that they would also benefit, because appropriate royalties would give authors a stronger motive to complete contributions on time, so that a book would be more up to date when published and, hence, more worthwhile buying.

JAMEs A. BarnetT

School of Biological Sciences,

University of East Anglia,

Norwich NR4 7TJ, UK

\section{An artist's eye}

SIR-As a corollary of recent correspondence (Nature 331, 10; 1988) about eye defects inferred from the paintings of El Greco, what are we to make of Lowry's matchstick men? Can van Gogh's vibrant use of colour be attributed to X-linked colour blindness, or Braque's cubism to possession of a compound eye by goldschmidtian macromutation? The preceding letter on the same page was headed: "God does not need science. .." — neither does the art critic.

Graham Wallis

117, Redfern Street,

Subiaco, WA 6008, Australia

SIR-The theory that El Greco had astigmatism was floated in the Paris Medical Chronicle in 1913; and it was taken up vigorously in the following decades by (largely German) oculists, who ingeniously countered - as did A.C. Dornhorst (Nature 329, 758; 1987) - the obvious objections that had been raised. These were discussed in a book I wrote 18 years ago (The World Through Blunted Sight, Thames \& Hudson, London, 1970), concluding that in the astigmatic artist "some such influence" was "just conceivable", because, as Nicholas Evans pointed out (Nature 331, 10; 1988), perceptual aesthetics can never be linked securely to organic anomalies, such as the shape of the eyeball.

When I first read Sir Peter Medawar's passage ridiculing the "nameless ophthalmologist" who surmised that El Greco was astigmatic (which you so applauded in your leading article 329, 472; 1987) I did draw his attention to the words I had actually used - labelling it as "this timehonoured (and indeed least probable) of all the theories propounding an organic influence on the artist's style". I was hoping that, if proved to be that nameless culprit, I had been misquoted to him, and so might be exonerated. His secretary confirmed that he had received my letter, but declared that he was "too unwell to deal with such correspondence".

\section{Park Square West,}

Patrick Trevor-Roper

Regents Park,

London NW1 4LJ, UK 